$16^{\text {th }}$ International Congress of Metrology, 09003 (2013)

DOI: $10.1051 /$ metrology/201309003

(c) Owned by the authors, published by EDP Sciences, 2013

\title{
Building Energy Efficiency: One metrological support example to vocational education and training
} \author{
Atanasov $^{6}$, Wim Geuvens ${ }^{7}$, Patrick Ampe ${ }^{8}$, Ludwig Cardon ${ }^{8}$ and Ivo Verjans ${ }^{9}$ \\ ${ }^{1}$ LCM - (LNE-Cnam), 29, Av. R. Hennequin, ZA Trappes - Elancourt, 78190 Trappes, France \\ ${ }^{2}$ Lycée Louis Vicat, 1, Rue Pierre Bourthoumieux, 46200 Souillac, France \\ ${ }^{3}$ NEOTIM, MDI - ZA Albitech, 54, Rue Gustave Eiffel, 81000 Albi, France \\ ${ }^{4}$ GRETA Quercy Rouergue, 5, Avenue du Maréchal Joffre, 12000 Rodez, France \\ ${ }^{5}$ SALPAUS Further Education, Teinintie 4, PO Box 207, 15201 Lahti, Finland \\ ${ }^{6}$ TU Sofia, Faculty of Engineering and Pedagogy, bd Burgasko Shosse, 8800 Sliven, Bulgaria \\ ${ }^{7}$ PISO, Alexianenweg 2, 3300 Tienen, Belgium \\ ${ }^{8}$ University College Ghent, Kluyskensstraat 2, 9000 Gent, Belgium \\ ${ }^{9}$ 2B-SAFE bvba, Muizenbempstraat 1, 3300 Tienen, Belgium
}

Jean-Remy Filtz ${ }^{1, a}$, Patrick Nicolas ${ }^{2}$, Jean-Philippe Marquié ${ }^{2}$, Marie-Christine Osmont ${ }^{2}$, Elian Coment ${ }^{3}$, Stephane Palaprat $^{3}$, Nathalie Lavaurs ${ }^{4}$, Françoise Villeneuve ${ }^{4}$, Gilles Fontange ${ }^{4}$, Ulla Pantsar ${ }^{5}$, Markku Ollinen ${ }^{5}$, Radostina Petrova ${ }^{6}$, Koycho

\begin{abstract}
The Energy Performance Diagnosis (EPD) has became a basic mandatory tool for addressing the needs of commercial real estate transactions. Within the framework of a European project (Leonardo da Vinci Partnership) an original contribution towards the European harmonization in education and training was undertaken. Among key expertises in various technical fields; Building design, Building physics, Energy consumption, Thermal and optical properties of materials, ... Metrology expertise has been identified as one of the competencies for structuring the frame of vocational education and training. This article gives an overview on main actions performed during the project.
\end{abstract}

\section{Introduction - Context}

The Energy Performance Diagnosis (EPD) is now mandatory before performing any commercial real estate transactions. This methodology is now progressively widely applied throughout Europe.

One question arising is how to implement education and training in order to answer the market needs and also for improving quality and truthfulness of the diagnosis.

Recently, within the framework of a European project "Thermovalorisations" (Leonardo da Vinci Partnership) an original contribution towards the European harmonization in education and training has been suggested and performed. Among different useful technical expertise; Building design, Building physics, Thermal and optical properties of materials, Energy consumption, measurement engineering , ... metrology expertise has been identified as one of the key competencies useful for structuring the frame of vocational education and training.

\section{Technical Objectives}

The project was structured around topics to be considered as three main goals to achieve $[1,2]$ :

\footnotetext{
${ }^{\mathrm{a}}$ Corresponding author: jean-remy.filtz@lne.fr
}

1) Making a survey: Comparison of the main European regulations and calculation methods according to the basic rules of the Energy Performance Diagnosis applied up to now in Europe.

2) Implementing the practical teaching of Energy Performance Diagnosis: Initiation to thermal measurements applied to buildings. Basic metrology rules with a protocol proposal for operating a thermal camera.

3) Improving the qualification of the students: Adaptation and optimization of the trainings course's content (initial or vocational training course) with the objective to certify or at least to upgrade or improve the students qualification.

The main objective is finally to establish harmonized tools enabling to carry out Energy Performance Diagnosis with more added value on the basis of new key competencies and new means such as a thermal camera.

Following a brief description of the main objectives of the project, this presentation is intended to give an overview on:

- $\quad$ implemented methodologies, 
- convergences and deviations in the different technical approaches,

harmonization constraints before approval of one unique European protocol,

contribution of the "Metrology expertise" to help structuring VET (Vocational Education and Training), some perspectives.

\section{Implementing the EPBD: Brief survey}

\subsection{European directives and requirements}

The Energy Performance Diagnosis (EPD) follows first from the implementation of the Energy Performance of Buildings Directive (EPBD) 2002/91/EC.

This first directive listed the main requirements for improving the energy performance of buildings:

- establishing harmonized methodologies for calculating the integrated energy performance of buildings (All buildings)

- applying of minimum requirements (new and existing buildings)

- defining and making available to the owner or by the owner to the buyer/tenant, the energy performance certificate of the building (energy certification of buildings)

- regularly inspecting boilers, air-conditioning systems and heating installations

Members states had to comply with this directive since 2006. [3]

In 2010, this Directive has been amended as Directive 2010/31/EU on the energy performance of buildings. With this new Directive, the objective was to clarify some points - introduction of new technical points such as "building elements", "building units" or "technical building" and to complete with new articles focused on issue of energy performance certificates, nearly zeroenergy buildings, legal aspects,....[4]

A correlation table between both Directives makes the understanding easier. For most of the articles listed, Members states have to comply with this new directive since 2012. However for "Nearly zero-energy buildings", Member stated shall ensure to be ready after 2020 .

Because the current scientific partnership ended before this new directive enters into force, the work of the group was performed according to the first Directive. The evolution between the Directives does not change the main conclusions.

\subsection{How Member States apply the current directive?}

The implementation of the EPBD by the different Member States is based on laws and decrees nationally published. Depending on very different regional climatic and local conditions in Europe (Figure 1), today rules and technical tools have been developed according to legal and incentive actions but are not fully harmonized. Therefore, before performing any comparison, it was agreed that the first part of this project should be dedicated to identify rules, tools and associated methodologies. This work was mainly focused on the calculation method and only performed for the countries represented within this partnership $[5,6]$.

Figure 1. Climatic and vegetation regions in Europe

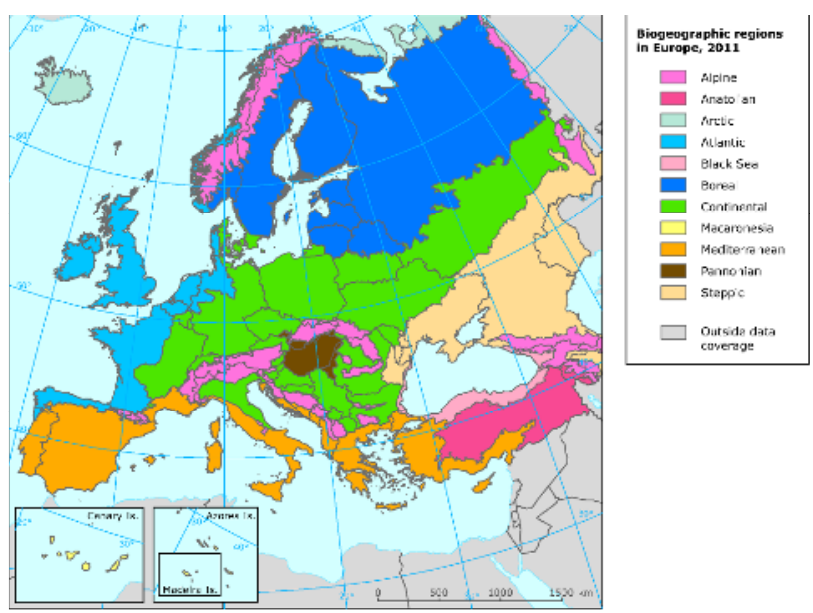

source: European Environment Agency (EEA) http://www.eea.europa.eu/legal/copyright

As a result of this investigation, the implementation of the EPD can be shortly summarized as follows:

\section{Belgium (Flemish Region)}

In Belgium, different methodologies are applied depending on the region; Flemish, Brussels Capital, Walloon. Since 2008 several certification experiences on both new and existing buildings have been performed.

In Flemish region, methodology, software and qualifications of the experts are different for new, residential or public buildings. For instance and to highlight; for the calculation of the energy consumption many default values are applied to increase the reproducibility. All heating, cooling, domestic hot water (only residential buildings), and lighting (only nonresidential buildings) and the production of renewable energy are taken into account. It is mandatory for qualified experts to use a specific software, which is an online system based on a central server. For the certification of residential buildings, an adapted calculation procedure was proposed.

\section{Bulgaria}

A general description of the calculation method has been made, keeping the basic requirements of the following European standards: EN 832, EN 13370, EN 13789 and should be available@ www.mrrb.government.bg. 
However, it appeared that no one software was easily identified and/or accessible. Nevertheless, during the project, the Bulgarian experts team used a software developed within the framework of academic Researches and was able to perform relevant simulations.

\section{Finland}

The calculation method is described in the building regulations. The main principles are in agreement with EN ISO 13790. More detailed modelling or simulation tools can also be used. Up to 10 main typologies of buildings can be considered. As reference value, the gross area of the building is taken into account. When establishing the certificate, the qualified expert has to make very detailed suggestions for saving energy.

\section{France}

French regulations propose labels for primary energy consumption and $\mathrm{CO} 2$ emissions, asset rating for residential buildings constructed after 1948 and operational rating for public buildings.

Energy consumption and calculation method: The 3CL methodology (Conventional Consumption Calculation in Housing) is a basic calculation satisfying to the minimum required. Method has been updated according to the French decree "Arrêté du 17-10-2012". More sophisticated methods (dynamic calculation methods) can also be applied. Only 3 main typologies of buildings (individual, apartment and public) are considered (Table 1).

More recently, a new thermal regulation (RT2012) came into force, with the objective to reduce the mean final energy consumption of housing and enabling to strengthen the implementation of the EPBD.

Table 1. EPC - Methodologies

\begin{tabular}{|c|c|c|c|}
\hline \multicolumn{4}{|c|}{ Enerqy Performance Certificate (EPC } \\
\hline & \multicolumn{3}{|c|}{ Building } \\
\hline 1 & \multicolumn{3}{|c|}{ Individual house } \\
\hline 2 & \multicolumn{3}{|c|}{$\begin{array}{l}\text { Apartment with individual heating } \\
\text { source }\end{array}$} \\
\hline 3 & \multicolumn{3}{|c|}{$\begin{array}{l}\text { Apartment with common heating } \\
\text { source or hot water production }\end{array}$} \\
\hline \multirow[t]{2}{*}{4} & \multicolumn{3}{|c|}{ Public } \\
\hline & \multicolumn{3}{|c|}{$>1948$} \\
\hline \multirow{5}{*}{$\begin{array}{l}\text { Sale } \\
\text { Rental* }\end{array}$} & \multicolumn{3}{|c|}{ Methodology } \\
\hline & Invoices & Invoices & Calculation \\
\hline & $1,2,3,4^{* *}$ & $3,4^{\star *}$ & 1,2 \\
\hline & $1,2,3$ & 3 & 1,2 \\
\hline & \multicolumn{3}{|c|}{$\begin{array}{c}{ }^{*} \text { except for overseas areas } \\
{ }^{* *} \text { of the last } 3 \text { years }\end{array}$} \\
\hline
\end{tabular}

\subsection{Conclusions}

The main identified conclusions of the working group were:
- Calculation of the surface area differs from a country to another one. Standard sizes/dimensions can include indoor or outdoor dimensions (gross or net area) of the house, stairs, garage, windows . These differences are significant and can be critical for analyzing/comparing the results

- Taken into account climatic differences in Europe, the comparison of climate data between countries becomes complicated . Therefore, considering the different software used, input data process produces substantial impact on the results.

- Depending on the country, calculation of the wall coefficients (Horizontal or vertical walls) for wall in contact with the floor is made differently. Thermal bridges are also managed differently.

- Conversion factor used for calculating the final energy in term of primary energy has a significant influence, impact on the results.

\section{Thermal Inspection: Smattering of knowledge}

\subsection{Added value of the project}

Teaching the basic rules of the EPD was a challenge for most of the staff of the schools in Europe. In France, very quickly and following a first experience lead on site by students, it was suggested to define protocols and complete the diagnosis with a thermal inspection by means of a thermal camera. This proposal was the basic innovative idea for setting up this project, with the main objective to share experiences and to finally make teachings for vocational education and training much more harmonized and consistent.

A set of European workshops were organized by the consortium with the support of experts in measurements and energy all along the project for studying $[7,8]$ :

- benefits and limits of a thermographic inspection

- how to usefully implement the diagnosis?, how to manage the risks assessment?

- definition of the elementary protocol when using a camera,

- cautions to be taken into account,

- how to structure an inspection report ? - what are the main contents to be dealt with?

- what is the field of application ? - limits of the protocol.

As a result of the different meetings, a set of raw procedures were agreed and can be summarized as follows: The thermal inspection was grasped as a safe, non-intrusive and relevant technique for :

- completing any other building diagnosis (i.e. EPD, blower door test)

- producing results in an easy way and very fast

- reporting relevant and useful observations

- identifying building defects not easily visible

- implementing the assessment of the risks

- preventing from any definitive damage

- helping for taking decisions

- helping for selecting optimized solutions 
- and finally contributing to save energy.

Practically, it was agreed this technique can help to identify:

- thermal insulation behaviour/quality

- thermal/cold bridging

- air leakage (openings, joints)

- damp walls, ceilings, corners,...

- energy systems type

- roller blind insulation

- discontinuity of insulation,...

- details of any structure,...

During the inspection and the measuring process, (See figure 2) the user of a thermal camera has to be aware of the most influence factors:

- emissivity of the different objects observed

- apparent reflected temperature by the objects,

- distance between the target and the camera,

- environment temperature,

- hygrometry,...

Figure 2. Thermal scene

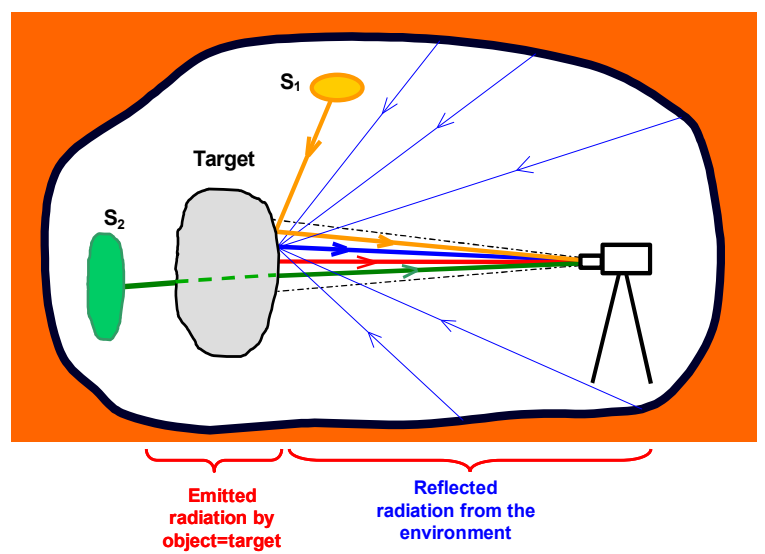

Using a thermal camera, the expert has to take care that emissivity is different from a material to another one and for each material (see examples figures 3, 4) ; emissivity can vary with temperature, wavelength, direction of observation and aspect of the surface (object).

Figure 3. Spectral directional emissivity of a white ceramic - LNE measurements

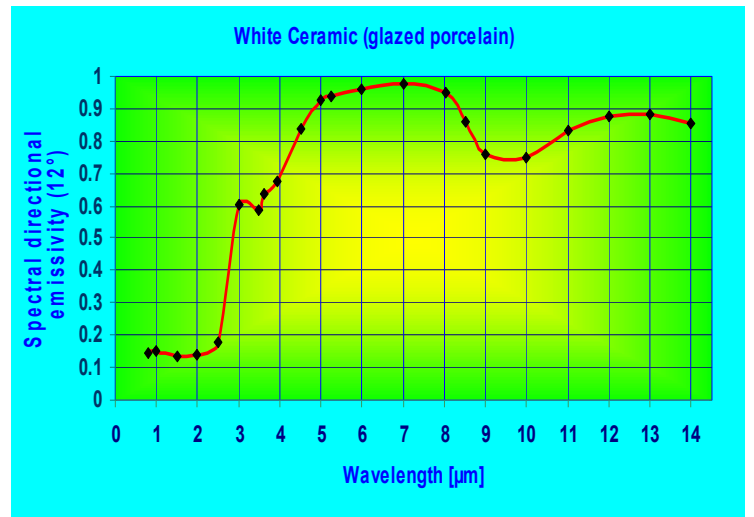

The expert can use an aluminium sheet and by adjusting the parameter emissivity to one, the environment temperature (commonly called Tref) can be estimated.

Figure 4. Spectral directional emissivity of aluminium LNE measurements

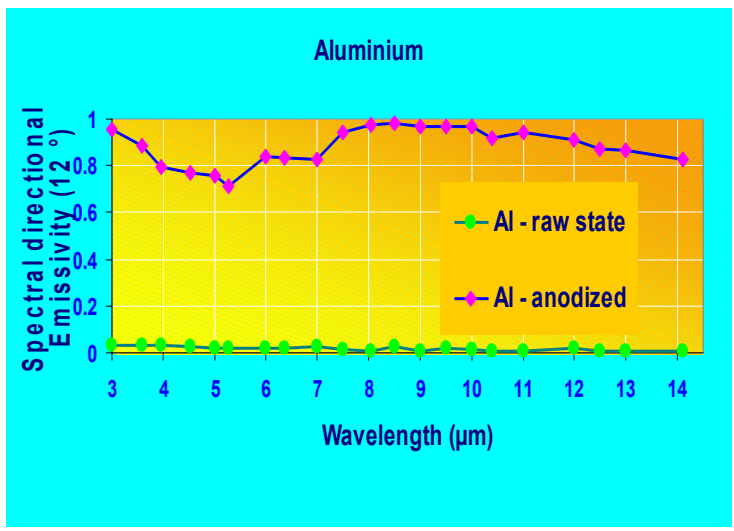

4.2 Basic procedures, Initial recommendations

Basic protocol of a thermographic inspection should be led as follows: As any efficient expert the main objectives are to:

- prepare the inspection in agreement with the needs of the client/customer,

- prepare all the tools useful for the inspection,

- plan the inspection accordingly to the weather conditions (outside) or to the use of the house (inside), - identify, check or confirm the quality of any structure or construction,

- give a reference for each IR and Visible image recorded,

- rank the observations (following technical interests),

- produce a relevant and useful readable report,

- make a "smart" presentation of the results to the client.

Inspection report: main contents suggested are: - location of the building,

- date of inspection,

- references of the building,

- references of the thermographer/energy auditor,

- thermal camera used, configuration,

- environment temperature,

- data selected (Emissivity, T ref, d, RH...)

- reference of the images (Visible, Infrared)

- comments, analysis,...

Thermography can or should contribute to strengthen the EPD. This additional investigation method should help to:

- identify defects, failures, leakages,

- propose solutions for reducing them,

- make recommendations for renovation and energy saving,

- support any economy analysis

\section{Adapting and optimizing Trainings}


For this chapter, the objectives of the European Vocational Training Curriculum to the job of EPD expert with a thermal camera were constructed and assembled according to four modules [9]:

\subsection{Module 1:Analysis and Energy Performances}

The goal of this module is to teach the basic knowledge enabling to understand how to perform an efficient and relevant EPD with the objective, when it is necessary, to suggest adapted renovation for saving energy. The expert has to propose solutions for improving the energy performance of the building. Additionally to the basic knowledge in science, building physics and heating systems, for this module it is highly recommended to manage the different current European directives, National laws and decrees and any regulation related to this topic.

\subsection{Module 2: Using a thermal camera}

The objectives of this module are first to understand the technology of a camera and then to perform relevant measurements taking into account some cautions. These recordings should help to identify insulation defects or heating system types and to bring added value to the EPD.

\subsection{Module 3: Performing a blower door test}

With this module, the objectives are first to identify leakages and any weak points of the building and secondly to compare measurements with current standards. Once again this methodology can help to bring added value to the EPD with other investigations techniques.

\subsection{Module 4: Advising the customers}

The objective of this module is to make sure that the student manages the basic rules for proposing the most appropriate solutions according to the demand and the need of the customer. He has to find the best compromise between to the economic, solution(s) to be applied, and energy criterions.

\section{Conclusions}

The project was very ambitious. The consortium was at least enabled to achieve the main following goals :

1) Realization of a brief survey related to the basic rules of the Energy Performance Diagnosis applied up to now in Europe; A first set of differences and limits have been identified and listed. This analysis has enabled to better manage the different calculation methods used in Europe and has indirectly contributed to suggest ways for harmonizing methodologies.

2) Using a thermal camera for detecting anomalies in buildings; Basic metrology rules with a protocol proposal for operating a thermal camera have been described. This work has allowed to propose additional methodologies and associated procedures for making useful decisions in renovation. Experts in Measurements and Energy have greatly shared their experiences and highlighted interests, and limits of the applied techniques.

3) Improving the qualification of the students with the objective that the junior expert can propose the most appropriate solutions according to the demand and the need of the customer; The experts and teachers have contributed to improve teachings accordingly to the current practical needs of this project - Vocational Education and Training.

\section{Acknowledgments}

This project was funded by the European Commission under the contract Nr 2009-1-FR-1-LEO04-07324-1. The consortium greatly thanks Philippe Bazzoli, President of CAPEB-47, for advices and support all along the project.

\section{References}

1. "From Energy Performance Diagnosis tests in houses and VET practices to new training and career", Final Report, DG Education and Culture, EC, Brussels, Belgium, (2011)

2. P. Nicolas, M.C. Osmont, E. Bascou, E. Coment, J.-R. Filtz, N. Lavaurs, J.-P. Marquié, S. Palaprat, “Thermovalorisations”, Working Report, (2011)

3. "Directive 2002/91/EC on the energy performance of buildings", Official Journal of the European Communities, (2003)

4. "Directive 2010/31/EU on the energy performance of buildings", Official Journal of the European Communities, (2010)

5. "Implementation of the Energy Performance of Buildings Directive", Country reports, EPBD Platform, European Communities, (2008)

6. "Implementation of the EPBD in Belgium, Bulgaria, Finland and France", Status in November 2010, Concerted action Energy Performance of Buildings, EC, Brussels, Belgium, (2010)

7. "EPD, Analysis of the methods, Results, Synthesis", Workshop Presentation, J.-R. Filtz, Rodez, France (2011)

8. D. Pajani «La Thermographie du Bâtiment, principes et applications $d u$ diagnostic thermographique », ISBN 978-2-212-13391-2, Eyrolles, (2012)

9. "Vocational Training Curriculum, Energy improvement of buildings", Greta Quercy Rouergue \& co-authors, Rodez Seminar, France,(2011) 